\title{
Corneal topography in keratoconus: state of the art
}

\author{
F. Cavas-Martínez ${ }^{1}$, E. De la Cruz Sánchez ${ }^{2}$, J. Nieto Martínez ${ }^{1}$, F. J. Fernández Cañavate ${ }^{1}$ \\ and D. G. Fernández-Pacheco ${ }^{1 *}$
}

\begin{abstract}
The morphological characterization of the cornea using corneal topographers is a widespread clinical practice that is essential for the diagnosis of keratoconus. The current state of this non-invasive exploratory technique has evolved with the possibility of achieving a great number of measuring points of both anterior and posterior corneal surfaces, which is possible due to the development of new and advanced measurement devices. All these data are later used to extract a series of topographic valuation indices that permit to offer the most exact and reliable clinical diagnosis. This paper describes the technologies in which current corneal topographers are based on, being possible to define the main morphological characteristics that the keratoconus pathology produces on corneal surface. Finally, the main valuation indices, which are provided by the corneal topographers and used for the diagnosis of keratoconus, are also defined.
\end{abstract}

Keywords: Placido disc, Detection system, Topographer, Diagnosis

\section{Background}

Despite earlier works in the study of the human cornea, the first morphological characterizations for clinical diagnosis were made through the simple observation of the external appearance of the ocular surface [1]. At the beginning of the XVII century, Schiener [2] proposed the first device for the characterization of corneal morphology, comparing the reflection of the image generated by the corneal anterior shape with different calibrated spheres. Later, in the mid-nineteenth century, the Goode keratoscope appeared and enabled a corneal examination using the lateral reflection of a bright square object projected onto the patient's cornea. Current corneal topography is based on the development of the Goode keratoscope by Antonio Placido da Costa [3], who developed it in 1880. This device was a variant that allowed a precise measurement of the anterior corneal surface through the projection of a circular plate onto the cornea. This plate, supported by a handle, contains a series of concentric rings and a hole in its center, allowing the doctor to see the reflected catoptrics

\footnotetext{
* Correspondence: daniel.garcia@upct.es

'Department of Graphical Expression, Technical University of Cartagena,

C/Doctor Fleming s/n, Cartagena 30202, Murcia, Spain

Full list of author information is available at the end of the article
}

image of the corneal surface of a patient located back to the light incidence. In the late nineteenth century, Javal performed the first qualitative analysis of the corneal morphology, joining Placido discs to his ophthalmometer and installing a viewer to obtain a wider field of the corneal image; he also proposed to photograph the image generated and to represent it on a diagram, which was aimed to analyze the variations in the curvature observed in the corneal surface. In 1896, Gullstrand [4, 5] presented the first quantitative assessment of the Placido discs' images. He joined the discs to his photokeratoscope and examined the corneal photographs using a microscope in order to calculate the corneal curvature by a numerical algorithm.

In the early 80 s, images were digitalized by hand and later computationally analyzed. Then, with the development of new computer equipment, the automation of this process appeared by capturing the image with a digital camera and its immediate computational analysis. The first fully automated corneal topographer was called Corneal Modeling System (CMS-1), developed by Computed Anatomy Inc. (New York, USA).

This article discusses the current technologies of corneal topographers and is aimed at: i) describing how they enable the main morphological measurements that 
produce the pathology of keratoconus on the corneal surface to be performed and ii) enumerating the main assessment indices provided by the topographers and used to diagnose keratoconus.

\section{Review}

\section{Corneal topography: current technologies}

Corneal topography is classically defined as a non-invasive exploratory technique to analyze both qualitatively and quantitatively the morphology of the cornea [6], enabling its geometric characterization and differentiating standard patterns from those potentially devastating for vision disorders caused by pathological ectatic conditions [7-11]. Current corneal topographers are based on one of these technologies: i) systems based on the light reflection on the cornea, ii) systems based on the projection of a slit light onto the cornea, and iii) systems based on the asymmetric reflexion of multicolor light-emitting diodes (LEDs).

\section{Systems based on the light reflection on the cornea}

Corneal topographers based on this technology, also called videokeratoscopes, are based on the application of the principles of convex mirrors' geometrical optics to an instrument in which the rings or Placido discs with known size and spacing are reflected on the anterior surface of the cornea. This image is initially captured by a digital camera and then processed by a computer. From identifying the edges of the rings, each topographer uses an algorithm reconstruction of the corneal curvature, which accuracy depends on how the programming architecture is defined. The so-called arc-step algorithms are the most used and are based on an iterative process that uses a sequence of arcs from point to point, covering the entire corneal region from the apex to the periphery. This process does not ignore data obtained in the previous step (step i-1) for obtaining current data (step i) [12].

Height and slope data derived from the radial curvature of the corneal surface are presented by topographers as corneal keratometric data of the entire surface by a series of maps that follow a color scale developed by the University of Louisiana [13] (Fig. 1).

- Cool colors correspond to flat curves and elevation values below the reference sphere (blue or violet colors).

- Mild colors correspond to medium curvature and elevation values equal to the reference sphere (green or yellow colors).

- Warm colors correspond to high curvature and elevation values above the reference sphere.

In addition, depending on the size of the Placido disc rings there are two commercial options [14]:
- Corneal topographer based on large diameter Placido disc rings. These devices are less susceptible to error associated with the misalignment between examiner and patient because they work at a large distance from the eye. However, because of this distance, it is possible to lose representative points because of the patient's facial morphology, produced by the shadow of the patient's nose and eyelashes.

- Corneal topograper based on small diameter Placido disc rings. These devices are most susceptible to alignment errors between the examiner and patient because they work in short distances, very close to the human eye. However, it mitigates the loss of information produced by patient's facial morphology, reducing the shadow that the nose and eyelashes could cause.

However, both systems have an important limitation that results from the use of internal algorithms that do not allow an accurate characterization of the corneal morphology in case of high levels of irregularity. In some cases, it is possible to obtain mistakes up to 4 diopters in corneas that present a very curved morphology, as it occurs in keratoconus disease [15].

\section{Systems based on the projection of a slit light onto the cornea}

These corneal topographers are based on the integration of a dual technology. The first process involves projecting a Placido disc, obtaining the mirror image by reflection and representing the curvature and refractive power, which is obtained by an arc-step algorithm. The second phase is the projection of a slit light onto the cornea. Due to the transparent structure of the cornea, and using Rayleigh scattering, it is possible to photograph it. These images will provide accurate data of corneal elevations for the entire anterior segment (see Fig. 2) [16].

Furthermore, this technology has two variants, depending on the spatial arrangement of the photographic system:

- System based on the principle of standard or normal photography. Its main feature is that the plane of the camera lens is located in parallel with the image. It means that only a small region is focused (the imaginary extensions of the film planes, the lens and the focal plane are parallel) (Fig. 3) [17, 18]. The most common system is the Orbscan (Bausch \& Lomb Incorporated, USA), which was the first commercial device that assessed the posterior corneal surface in a non-invasive and quick way. This system provides different maps of the anterior and posterior corneal surfaces, and also pachymetric data. However, several authors from scientific 


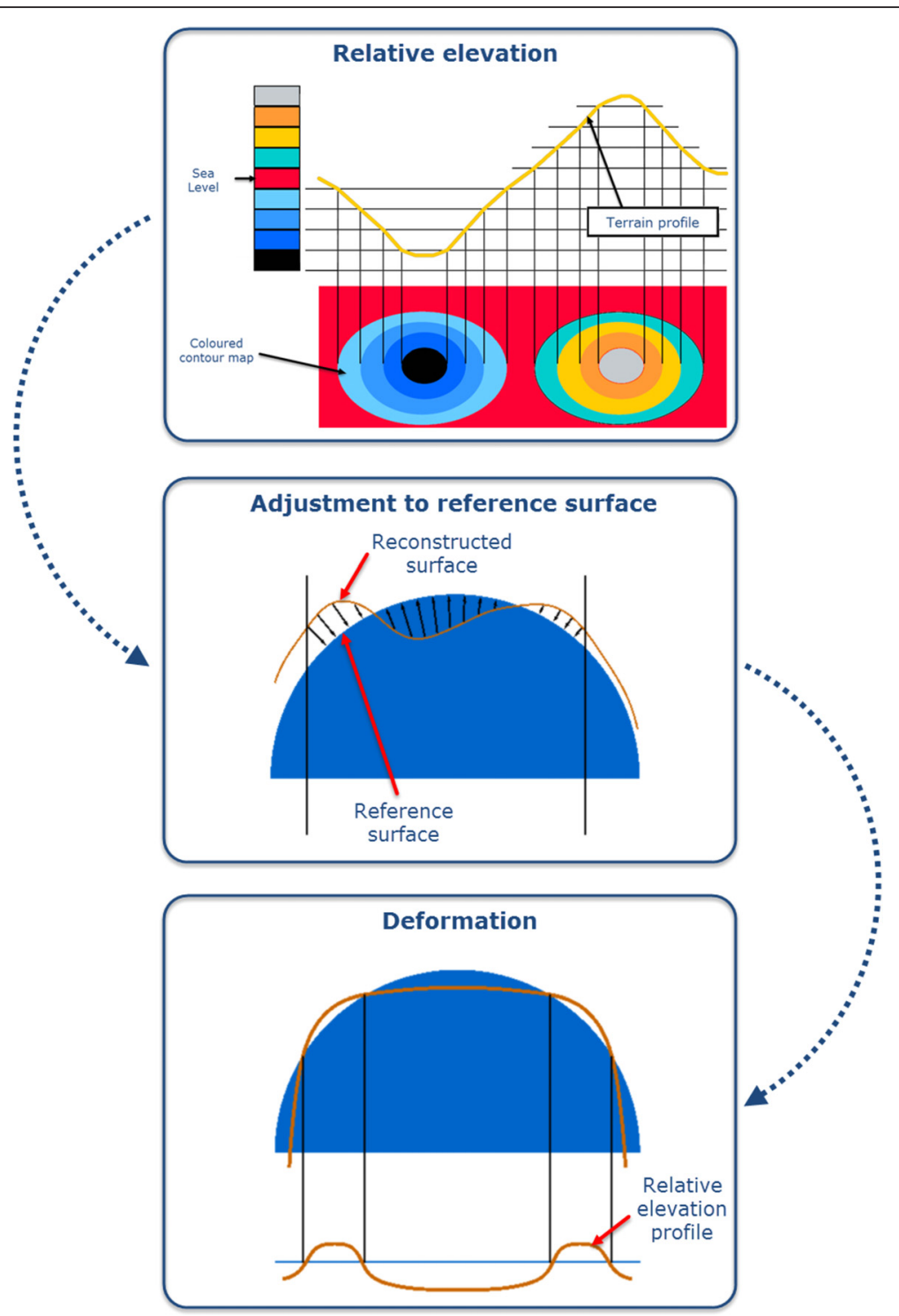

Fig. 1 Corneal topography

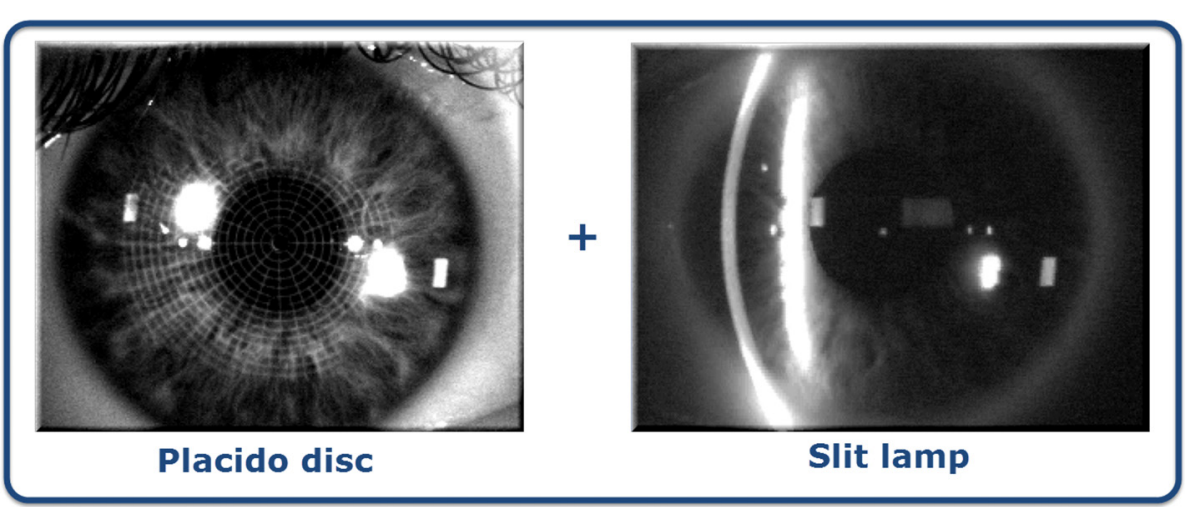

Fig. 2 Systems based on the projection of a slit light on the cornea 


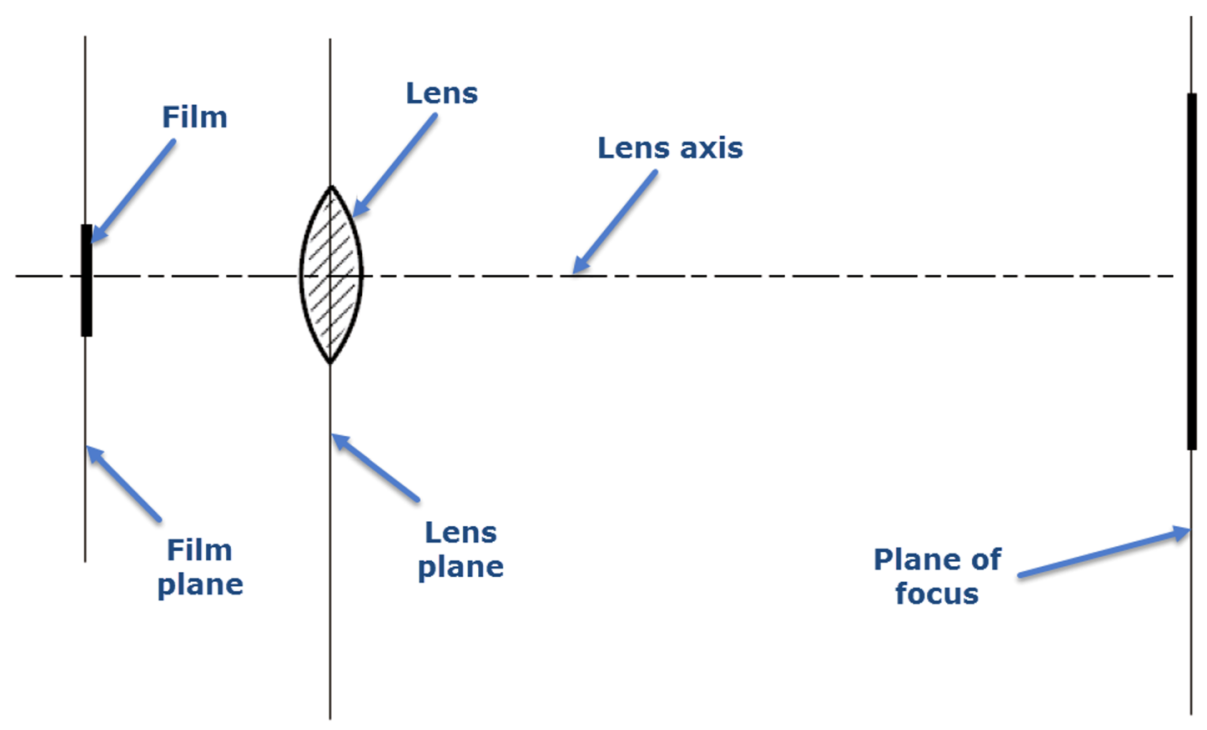

Fig. 3 Principle of standard photography

literature present a strong controversy due to the reliability of the measurements performed by this device on the posterior surface, and also the limited repeatability $[13,19,20]$.

- Systems based on the principle of Scheimpflug photography. Its main feature is that the plane of the camera lens is placed sideways to the image. The imaginary extensions of the planes of the film, the lens and the focal plane are not parallel (Fig. 4) [18], and therefore the focused region is increased and the image sharpness is improved [10,21, 22]. The main commercial systems based on this principle are Pentacam (Oculus, USA), Galilei (Ziemer, Switzerland) and Sirius (CSO, Italy), which offer repeatable measurements of the corneal curvature and other anatomical measurements of the anterior segment. However, several authors question the degree of concordance between the measurements provided by these devices [23-25].

\section{Systems based on the asymmetric reflexion of multicolor LEDs}

These systems are based on the same optical principle than convex mirrors, but in this case the emitters are multicolor LEDs.

More specifically, this system uses a panel formed with an asymmetric distribution of more than 700 LEDs in colors red, yellow and green, which reflexion on the cornea

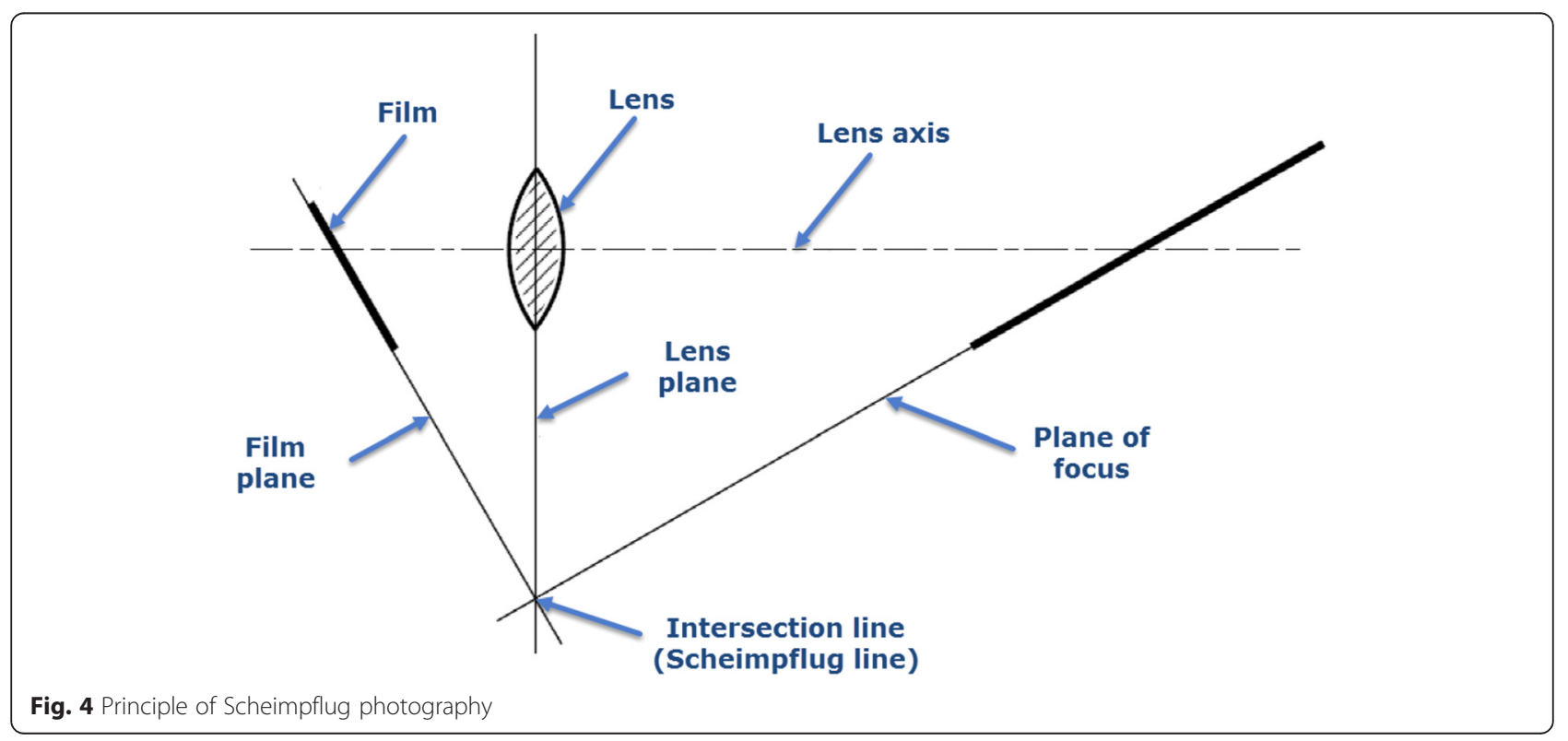


provides a more accurate reconstruction of the corneal surface if compared with the lack of detail obtained when projecting a monochromatic light [26-28].

The main novelty of this system is the use of a unique reconstruction algorithm for the reflexion of each LED projection; this one being independent of the algorithm used for the other LED projections. This new approach has one main difference with respect to the systems previously described where the rest of them were based on the projection of a monochromatic light and use the same reconstruction algorithm for all the points of the corneal surface. Moreover, the new device permits redefining at the local level, every three points identified in the corneal surface, its curvature or elevation.

The unique commercial equipment based on this principle and currently available is the Cassini color LED Corneal Analyzer (i-Optics, The Netherlands) [26-28].

\section{Errors}

The use of previously described corneal topography technologies involves the generation of errors during the acquisition process and data handling, and can be distinguished between intrinsic and extrinsic errors.

\section{Intrinsic errors}

These errors are derived from the equipment itself, so the measurements performed almost always include errors, as noise. This should be taken into consideration in data handling and data analysis [29-34]. Thus, devices show limitations derived from the measurement errors and digitalization [35], and also due to the internal algorithms making assumptions, rounding or approximation hypothesis. For this reason, it is necessary to consider that high levels of irregularity in corneal morphology, as a result of ectatic corneal pathology, could lead to a poor representation of the actual geometry of the cornea $[15,31,34,36]$. In the case of insufficient data for the reconstruction of the corneal surface, these are internally provided by the device, which can lead to a biased characterization [36-38].

All these factors cause additional contamination of the measurement. In order to fix this problem, a preprocessing stage of the signal could be included, allowing the location and correction of the areas with high interference or incomplete data. But all the proposed solutions have a high computational cost, and are difficult to implement in real time, for instance, the use of bi-dimensional Gabor filtering to detect areas without directional pattern in the rings $[39,40]$ or the application of image processing techniques to improve data acquisition from areas with incomplete information [41].

Recent studies show that such errors can be reduced by new corneal topographers without a significant influence in data acquisition and processing [35, 42], so ophthalmologists accept the existence of these errors, which are considered negligible when we consider the clinical advantage that these topographers provide for the diagnosis of ectatic corneal diseases.

\section{Extrinsic errors}

These errors are derived from how the clinician or examiner handles the topographer, mainly during the approach and alignment of the system with the examined patient's eye, and may lead to incorrect levels of astigmatism if wrongly performed. It can also fail due to an incorrect placement of the spatial center of the rings or the central crowns of Placido discs since curvature values are extremely sensitive to the misalignment or the fixation of the patient; thus, the accuracy of the measurements obtained by the device depends on good centering, alignment and focus by the clinician or examiner [43, 44].

Moreover, extrinsic errors derived from the quality of the tear film are also produced. Poor quality of the tear film together with the breakage in the distribution of the tear induce the appearance of anomalous data in the measures because of the distortion of the image of the Placido discs, which lead to the emergence of falsely irregular corneal spatial regions [45]. Also, alterations in the transparency of the cornea due to an ablation procedure as well as the existence of corneal scars or leucomas, may compromise the transparency of the cornea, resulting in the obtaining of erroneous pachymetric maps or altered curvatures maps, which may be confused with ectasia or irregular astigmatism [46].

Another source of error is the execution of the scanning process together with other clinical examination procedures. Recent research works have confirmed a significant variation in corneal parameters after instillation of different anesthetic eye drops. It has also been demonstrated that the dramatic reduction of the intrasession repeatability of measurements in this context, so this should be considered in order to avoid inappropriate clinical decision making [47].

Finally, another extrinsic error is derived from inadequate patient cooperation; a good eyelid opening during the examination is needed in order to obtain an adequate coverage of the corneal surface. In addition, the examiner should avoid the possible obstruction of the visual field resulting from patient's eyelashes [48].

Current devices incorporate new technologies that provide an index to quantitatively assess the quality of each measurement based on the extrinsic errors described above. The measurement process will require repeats in those cases in which data does not meet a minimum standardized reliability criterion, or when it is not possible to do a correct interpretation of data for a proper diagnosis [43]. 


\section{Topographic analysis of keratoconus disease}

Keratoconus is morphologically characterized on the anterior corneal surface by a cone-shaped protrusion $[49,50]$, generally eccentric with an inferior-temporal spatial orientation, which is physically interpreted as an area higher than the curve of the best adjustment surface in the elevation maps, and as an area more curved in the curvature map. This characteristic pattern of the corneal architecture is employed as a marker of severity of the keratoconus: keratometry values of 46 diopters or higher should be considered by the ophthalmologist as a sign of keratoconus disease [51]. The most frequent location of this corneal focal curvature is the mid-periphery region, both in the inferior-nasal and inferior-temporal quadrants. Specifically, the paracentral region comprises $72 \%$ of cases, and approximately $25 \%$ of those cases are included in the central region. Therefore, $97 \%$ of keratoconus cases are located in the central and paracentral corneal regions, where presence of the protrusion in the peripheral region is being considered unusual [52-54].

The manifestation of this protrusion in the surface of the anterior shape generates a structural weakness in the cornea, which also implies a change in the morphology of the posterior corneal surface, showing an increased curvature when comparing with a healthy cornea, even during incipient stages [55]. There are several studies that have characterized and clinically evaluated the geometric correlation between anterior and posterior surfaces, both in healthy corneas $[56,57]$ and corneas with keratoconus disease [58]. Other studies have evaluated the curvature and the elevation of the posterior surface of the cornea in eyes with keratoconus to quantify this increase and assess whether these changes can be used as clinical tools for the diagnosis of subclinical keratoconus $[55,59]$.

Corneal topographers provide different maps to represent the measurements that characterize the surface of a keratoconic cornea. The ophthalmologist has to decide what scale should be considered in each case in order to get the best information from a clinical point of view [47]: the absolute scale gives the entire diopter range that the topographer can measure on a color scale, so it loses sensitivity to small changes whereas the relative scale adjusts the dioptric measurement range for each cornea, so it is sensitive to small changes and is more appropriate for a customized analysis of corneal morphology $[7,9,52,60]$.

Current topographers provide, among others, the following maps:

- Curvature keratometric maps. They provide information about curvature at each point of the corneal surface, and can be axial (or sagittal) and tangential (or instant). Although both types report information about focal curvature, there exist significant differences between them $[9,61]$ :

- Sagittal map. This type of map fixes the curvature centers on the optical axis and considers the corneal surface to have a spherical geometry, achieving a marked overall smoothing of the corneal periphery. This results in a larger and more peripheral curving area than the actual area of the cone. However, this consideration is erroneous and only true in the paraxial approach since the cornea has a spherical surface.

Therefore, it distorts the true picture of the cornea and provides quantitatively inaccurate values $[9,47,61,62]$. However, this map is useful for qualitative assessment through colors due to it softening the geometric contours of the cornea and facilitating the interpretation of the results by less-experienced users [47] (Fig. 5).

- Tangential map. This map does not assume the spherical morphology of the cornea and therefore, the tangential curvature algorithm reconstructs the corneal surface by means of local curvature radii whose centers are not located on the optical axis. This map represents more accurately the curvature of the peripheral corneal region (Fig. 6) [47]. This map also shows a high sensitivity to data obtained, being suitable for monitoring the conical shape of the ectatic disease; however, it is less intuitive than the axial map and its interpretation may be more complex.

- Elevation maps. These maps do not represent data directly measured by the corneal topographer, but are obtained by comparing the reconstruction of the anterior or posterior corneal surface to the best fitted surface, typically a sphere, a toroid, a revolution ellipsoid or a non-revolution ellipsoid.

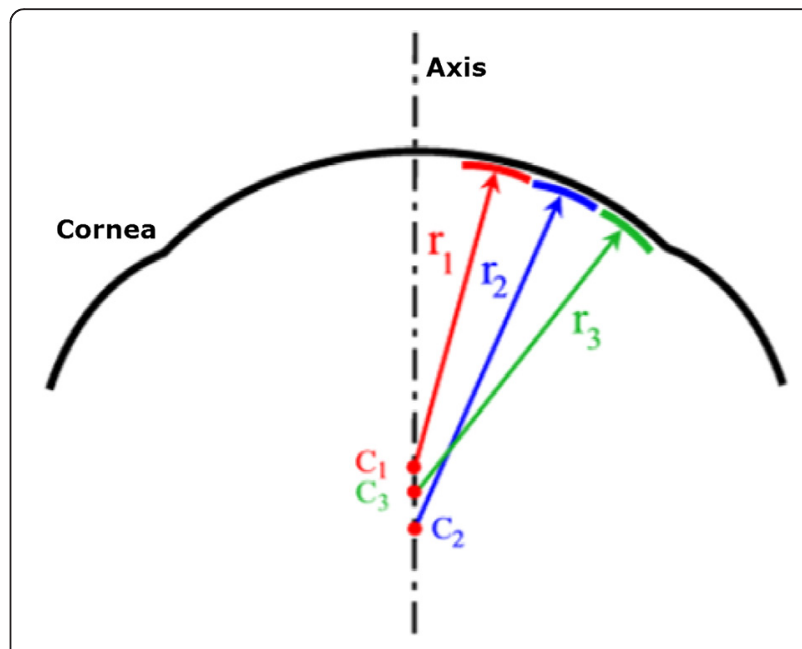

Fig. 5 Geometric reconstruction of the corneal sagittal map 


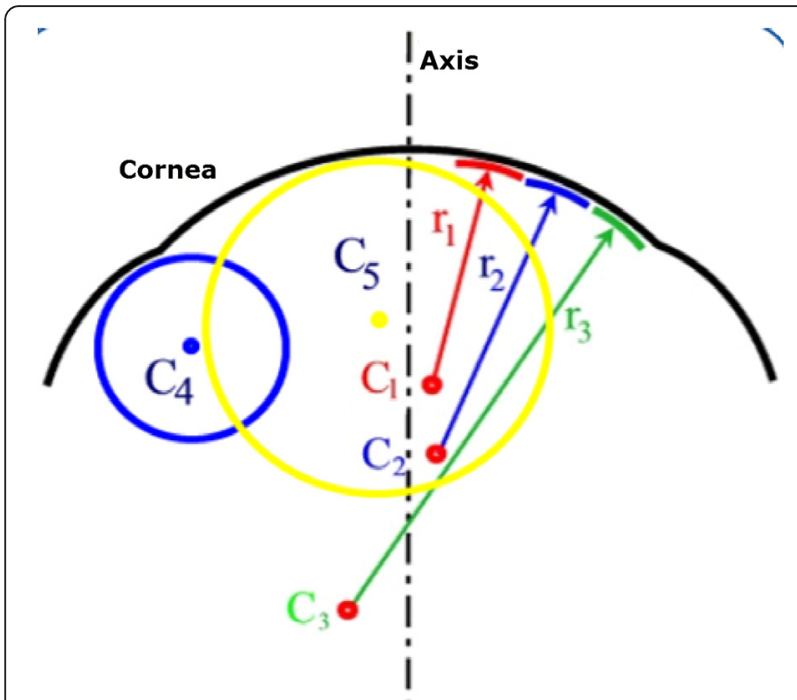

Fig. 6 Geometric reconstruction of the corneal tangential map

The difference between both surfaces is provided by altimetry data that correspond to the elevation maps (Fig. 7) [14, 63, 64]. In addition, typical dimensions of the reference surface are around $8 \mathrm{~mm}$ in diameter, so scenarios that may influence the data acquisition process, such as shadows generated by eyelashes, are avoided [48]. These maps present several advantages: i) data are presented quantitatively in $\mu \mathrm{m}$, so they are highly accurate and have a high sensitivity to small changes that can occur in the corneal morphology as a result of keratoconus [9], ii) topographers allow the selection of the best suited surface to perform the elevation map, which results in a sensitivity increase of the clinical diagnosis. This map provides, for both corneal surfaces, the elevation of the corneal apex, the elevation of the minimum thickness point [64] and the elevation of the center of the central region [65]. As the posterior surface is not altered by the excimer laser photoablation or by the generation of the corneal flap, nor is adulterated by the hyperplastic effect of corneal epithelium, data from the posterior surface could be very useful for the clinical diagnosis of keratoconus [9, 13, 47, 65, 66].

- Thickness map. This map does not show data directly measured by the corneal topographer, but must be precisely reconstructed using the anterior and posterior corneal surfaces. This map gives information of the minimum thickness point and its position on the center of the cornea. This point is essential for maintaining the corneal structure and determining the progressive thinning of the cornea when the severity of keratoconus progresses [67, 68]. Some studies in the scientific literature evaluate the pachymetric profile from the center to the periphery depending on the average thickness of the concentric rings separated by $0.1 \mathrm{~mm}$ and centered at the minimum thickness point. This profile presents a more abrupt change in a pathological stage than in a healthy scenario $[61,69,70]$.

\section{Indices for assessing irregularities in the corneal surface}

The main use of corneal topography is the generation of indices that allows quantification of the level of irregularity of the corneal morphology, at a local or general level. From these data, there are clinical studies that have tried to determine cut-off values to distinguish between normal and pathological corneas as well as to define several severity degrees of keratoconus. However, the main problem with these indices lies in the fact that each index has a high degree of specificity for the corneal topographer for which it has been developed, and cannot be directly extrapolated to other corneal topographers. In scientific literature, there are numerous indices used for the diagnosis of keratoconus, which are known as univariate or multivariate detection systems. Depending on the analysis approach, it could be a single index

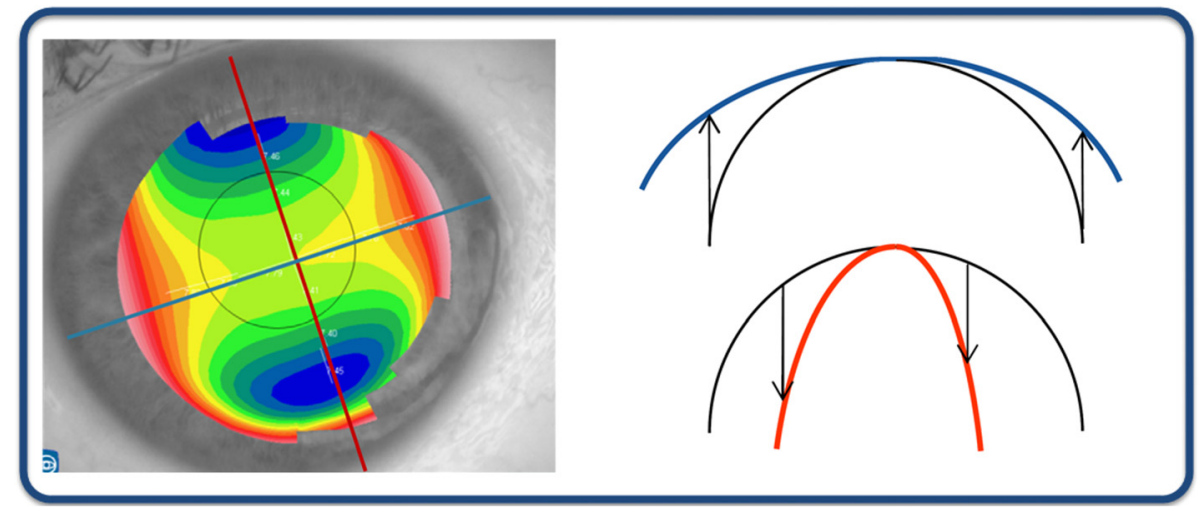

Fig. 7 Altimetry elevation map 
or a combination of indices that allows the interpretation of the main topographic characteristics of the keratoconus disease.

\section{Univariate quantitative detection systems}

- Simulated keratometry (SIMK). This index provides information on the diopter power of the flattest and most curved meridians in the so-called useful region of the topographer (ring diameters between 3 and $9 \mathrm{~mm}$ ). Numerically, it is expressed as $\mathrm{K} 1$ and $\mathrm{K} 2$, and the difference between the two values provides a quantitative value of corneal astigmatism. A clinical study provided SIMK mean values of $43.53 \pm 1.02 \mathrm{D}$ for a group of normal corneas, and established a cut-off value for keratoconus group twice the value of the standard deviation of the control group [14, 71].

- Surface asymmetry index (SAI). It is an index that indicates an average value of the power differences between the points spatially located at $180^{\circ}$ from 128 equidistant meridians. A radially symmetrical surface has a value of zero, and this value increases as the degree of asymmetry is greater [14, 72, 73].

- Surface Regularity index (SRI). It is a local descriptor of regularity in a central area of $4.5 \mathrm{~mm}$ of diameter (it comprises the central ten rings of Placido Disc). SRI quantifies power gradient differences between successive pairs of rings in 256 equidistant semimeridians. It correlates well with the value of visual acuity ( $p=0.80, P<0.001$ ), assuming the cornea as the only limiting factor for vision. A normal cornea presents SRI values below 0.56 (this value would be 0 in a perfectly regular cornea) [14, 52, 72].

- Central Keratometry (K Central). This is the average value of corneal power for the rings with diameters of 2, 3 and $4 \mathrm{~mm}$. Values below 47.2 $\mathrm{D}$ are considered normal, while values between 47.2 and 48.7 D are considered probable keratoconus. Values above 48.7 D are clinical keratoconus [14, 74].

- Predicted Corneal Acuity (PC Acuity). It quantifies the optical quality in Snellen units (with a range of $20 / 10$ to 20/200) in the central zone of the cornea with $3 \mathrm{~mm}$ diameter [72, 75].

- Inferior-Superior Value (I-S). This index is defined as the power difference between five points of the inferior hemisphere and five points of the superior hemisphere of the corneal region located at $3 \mathrm{~mm}$ from the corneal apex at spatial intervals of $30^{\circ}$. A positive value indicates higher inferior curvature while a negative value indicates higher superior curvature. I-S values between 1.4 and $1.8 \mathrm{D}$ are defined as cut-off points for suspected keratoconus and I-S values higher than $1.8 \mathrm{D}$ as cut-off points for clinical keratoconus [74, 76].
- Average corneal power (ACP). This index indicates an average power value of various points in the central corneal region [74].

- Topographic irregularity (IT). It is the root mean square (RMS) value of the difference between the actual topography data and a geometric reference surface, which in this case is a spherical cylinder that best fits the corneal surface of the study [72].

- Skew of steepest radial axis (SRAX). It measures the angle between the more curved superior semimeridian and the more curved inferior semi-meridian. The more curved semi-meridian for each hemisphere is determined by averaging the powers of the rings from 5 to $16 \mathrm{~mm}$ diameter. The smallest angle between these semi-meridians is subtracted from $180^{\circ}$, and the result, in degrees, is the SRAX index. A value greater than $20^{\circ}$ is considered indicative of keratoconus, but due to the high dispersion of values in some astigmatic corneas, this value is only valuable if corneal astigmatism is greater than $1.5 \mathrm{D}$ [72].

- Corneal uniformity index (CU). It corresponds to an index that quantifies the distortion uniformity in the central area with $3 \mathrm{~mm}$ diameter. It is expressed as a percentage, that is, a value of $100 \%$ indicates that the cornea has a perfect consistency [75].

- Irregular astigmatism index (IAI). It is a measure of dioptric variables along each semi-meridian, which is normalized by the mean power of the cornea and the number of measured points [72, 74].

- Apex curvature (AK). It corresponds to an index that provides a value of the instantaneous curvature in the corneal apex. Values below $48 \mathrm{D}$ indicate normality, values between 48 and $50 \mathrm{D}$ are suspect, and values above $50 \mathrm{D}$ denote abnormally high curvature [72].

- Asphericity coefficient (Q). It is an index that describes how the corneal curvature changes from the central region to the peripheral region. Its value depends on the diameter of study, so it is necessary to indicate it when expressing a particular index [75]. The most common parameter used in literature is Q. In a natural or non-pathological stage, the section of the cornea is a prolate ellipse with an average $Q$ value setting $-0.20 \pm 0.12$ [77, 78]. This is physically interpreted as the cornea is more curved in the center than at the periphery. For keratoconic corneas, Savini et al. [24] reported Q values in the corneal region that comprise a $8 \mathrm{~mm}$ diameter of -0.84 for the anterior surface and -1.10 for the posterior surface. Another study comprising the same diameter and the same corneal region reports $\mathrm{Q}$ values of the anterior surface between -0.65 and -1.18 and between 
-1.17 and -0.66 for the posterior surface [79], according to the severity of keratoconus using the Amsler- Krumeich classification [80, 81]. The values of asphericity describing the geometry of the cornea showed that the central and paracentral regions are more curved than the peripheral region, and this difference is greater in keratoconus disease than in normal corneas. Moreover, these values are higher when the severity of keratoconus increases. However, due to asphericity is measured in the central region $(4-5 \mathrm{~mm})$, if protusion is located in the peripheral region, the topographer may provide normal or even positive values of asphericity. Therefore, this quantitative index is not very specific for the diagnosis of keratoconus, and must be considered in relation to the apex position in the cone.

- Effective refractive power (EffRP). Index averaging the power in the central area of $3 \mathrm{~mm}$ diameter. It considers Styles-Crawford effect [75].

- Corneal Irregularity Measurement (CIM). This is a numeric index representing the degree of irregularity in the morphology of the corneal surface. It quantifies the standard deviation between the corneal surface and the best-fit reference surface, which in this case is a toric surface. High values of this ratio indicate a greater possibility of the cornea to present a pathology related to a morphological abnormality. A healthy cornea has CIM values from 0.03 to $0.68 \mu \mathrm{m}$, while a value from 0.69 to $1 \mu \mathrm{m}$ is considered as suspect or normal limit, and a value from 1.10 to $5.00 \mu \mathrm{m}$ as pathological or unusual [14].

- Analyzed area (AA). It is the ratio of the data area interpolated by the area circumscribed by the outermost peripheral ring [72, 74].

- Mean toric keratometry (MTK). This index is obtained from the data of the elevation map of the cornea. It compares and analyzes the elevation values of the cornea calculated by means of the best adjustment to a toric reference surface. If the MTK value is high, the cornea acquires a geometrical behavior similar to a toric surface, which is clinically interpreted as a major probability of suffering ectatic alterations. The MTK index follows a Gaussian probability distribution, with an average value of $44.5 \mathrm{D}$, and the range defined for the values from $41.25 \mathrm{D}$ to $47.25 \mathrm{D}$ contains $96 \%$ of the population. [82].

- Centre Surround index (CSI). It is an index that quantifies the average power difference between the central zone of $3 \mathrm{~mm}$ diameter and a half-peripheral ring that is 3 and $6 \mathrm{~mm}$ diameters [72, 74].

- Different sector index (DSI). It is an index that quantifies the average power difference between sectors of $45^{\circ}$ with the highest and lowest power $[72,74]$.

- Opposite industry index (OSI). This is another index indicating in terms of average power difference between opposing sectors of $45^{\circ}[72,74]$.

- Calossi-Foggi Apex curvature gradient (ACG). This index quantifies the average difference per length unit of the corneal power in relation to the apical power. Values greater than $2 \mathrm{D} / \mathrm{mm}$ can indicate keratoconus, between 1.5 and 2 suspect, and less than 1.5 is considered a normal value [72].

- Calossi-Foggi Top-Bottom Index. It is a vertical asymmetry index similar to I-S value, but this indicates the difference in terms of average power between the superior area and an inferior area. This last is determined according to a major probability of presence of the cone. A positive value means that inferior area is more curved, and vice versa. A value less than 1.5 is considered normal, a value between 1.5 and 2 considered suspect, and values greater than 2 are considered abnormal [72].

- Orbscan surface irregularity. It is a coefficient calculated from the standard deviations of the mean curvature and mean astigmatism in the zones comprised between the center rings of 3 and $5 \mathrm{~mm}$ diameter. Values greater than 1.5 in the area of $3 \mathrm{~mm}$ and/or 2 in the area of $5 \mathrm{~mm}$ are indicative of high irregularity. This value is not specific for keratoconus, but merely indicates irregularity [72].

\section{Multivariate quantitative detection systems}

The diagnosis of keratoconus has been facilitated by the application of different quantitative detection systems using multivariate combinations of the topographic indices described above. Each topographer incorporates a different strategy and it is essential for the user to understand the sensitivity and specificity of each software. These systems can be:

- KISA\%. It is calculated from four indices: Central $\mathrm{K}$, SIMK, I-S and SRAX. It is a very effective index in the identification of keratoconus, but may have a significant number of false negatives in the clinical diagnosis and in incipient keratoconus cases, which can suppose a major risk in its use as a screening tool in refractive surgery. A value between 60 and 100 is indicative of suspected keratoconus, while a value greater than 100 is diagnostic of keratoconus; for this last value the KISA index has a high sensitivity and specificity $[14,72,83]$.

- Chastang method. Combines SDSD and Asph indices, developing a primary decision tree. This method is not applicable for defining the grade or 
severity of keratoconus, so incipient or early cases are not identified [72, 84].

- Belin/Ambrósio Enhanced Ectasia Display III (BAD III). This method combines the following nine parameters: anterior elevation at the minimum thickness point, posterior elevation at the minimum thickness point, change in anterior elevation, change in posterior elevation, corneal thickness at minimum thickness point, location of thinnest point, pachymetric progression, Ambrósio relational thickness and Kmax. The BAD III index provides individual information for every parameter and then performs a discriminant analysis combined with a regression of the nine indices, which permits the discrimination between healthy and keratoconic corneas [85].

- Keratoconus severity index (KSI). This index is also known in the Ophthalmology field as the SmolekKlyce method [71]. This multivariate system calculates the severity of keratoconus, which possibly distinguishes between a healthy cornea, a suspected keratoconic cornea and a cornea with keratoconus. The algorithm used is based on a neural network with 10 topographic indices as inputs. A KSI value $<15 \%$ is considered normal, values between 15 and $30 \%$ as suspected keratoconus, and above this value is considered subclinical keratoconus [14, 71, 72].

- Keratoconus Index (KCI). This is a method commonly known in the Ophthalmology field as the Klyce-Maeda method [86]. This method can differentiate a healthy cornea from a keratoconic cornea, and also distinguish between keratoconus developed in the central or the peripheral regions.

- Keratoconus prediction index (KPI). It is calculated by a combination of 8 topographic indices and uses a linear discriminant function. These indices are: Sim K1, K2 Sim, UPS, DSI, OSI, CSI, IAI and AA. A value greater than 0.23 is suggestive of keratoconus. In a validation group of a hundred eyes with different clinical conditions, this method showed a sensitivity of $68 \%$ and a specificity of $99 \%$. The KPI multivariate method along with DSI, OSI, GSI and Sim K2 indices were implemented using an expert computational algorithm based on a binary decision tree [72].

- PathFinder Corneal Analysis. It is a system which uses three different indices (CIM, Q and MTK) for detecting the morphological alterations that corneal topographies present. In the case of a normal cornea, the three indicators give values within normal limits. In the case of molding due to contact lenses, there is also a corneal distortion known as pseudo-keratoconus, where the CIM index is outside the normal range, but the other two indicators are within normal limits. In the case of a subclinical keratoconus, central curvature values and inferiorsuperior asymmetry cause both the CIM and the MTK to have abnormal values, while asphericity remains normal or borderline normal. In the case of keratoconus, the three indicators are outside the bounds of normality $[14,87]$.

- Rabinowitz and McDonell Index. It is one of the first multivariate indices based on the combination of the numerical values supplied by the I-S index, the value of the center, $\mathrm{K}$ and the difference of the central $\mathrm{K}$ between both eyes of the patient. This multivariate system combines the information obtained from the central curvature values with the inferior-superior asymmetry values as diagnostic parameters of keratoconus that can occur in both central and peripheral regions. However, this indicator is unable to quantify the amount of irregular astigmatism associated with the keratoconus disease [72, 76].

\section{Conclusions}

One important characteristic of the corneal topography is the possibility of generating a series of indices that quantify the level of irregularity of the corneal morphology at the local or global level. Some previous clinic studies have used these data to calculate the cut-off values that permit the discrimination between normal and keratoconic corneas as well as to define several stages or grades of keratoconus. However, these indices present two problems. On one hand, each index has a high grade of specificity for the corneal topographer for which it has been developed, not being possible to extrapolate it directly to other corneal topographers. On the other hand, univariate or multivariate indices do not provide clinical parameters for the diagnosis of keratoconus with $100 \%$ sensitivity and specificity. For that reason, and after reviewing the existing technologies and topographic indices described in the literature, the present paper proposes a new concept for the integral morphological analysis of the cornea in which several topographic indices are analyzed simultaneously with the aim of providing the most exact and reliable clinical diagnosis.

\section{Competing interests \\ The author(s) declare(s) that they have no competing interests.}

\section{Authors' contributions}

All authors drafted the manuscript. FCM wrote the background section, as well as the part which describes the current technologies used for corneal topography. EDS wrote the errors (intrinsic and extrinsic) section. DFP wrote the abstract, the section for the topographic analysis of keratoconus disease, the final conclusions and also submitted the manuscript. FFC and JNM wrote the section which describes the indices for assessing irregularities in the corneal surface: FFC was in charge of the univariate quantitative detection systems and JNM was responsible of multivariate quantitative detection systems. All authors read and approved the final manuscript. 


\section{Author details}

'Department of Graphical Expression, Technical University of Cartagena, C/Doctor Fleming s/n, Cartagena 30202, Murcia, Spain. ${ }^{2}$ Faculty of Sports Science, C/ Santa Alicia s/n, Santiago de la Ribera, 30720 Murcia, Spain.

\section{Received: 15 September 2015 Accepted: 14 February 2016} Published online: 22 February 2016

\section{References}

1. Güemez-Sandoval E, Güemez-Sandoval JC. Representaciones anatómicas de ojo a través de la historia. De Hipócrates a Mollineti. Revista Mexicana de Oftalmologia. 2009;83(3):186-91.

2. Gutmark R, Guyton DL. Origins of the keratometer and its evolving role in ophthalmology. Surv Ophthalmol. 2010;55(5):481-97.

3. Nover A. 100 years of ophthalmology. Fortschr Med. 1982;100(47-48):2222-7.

4. Sulek K. Prize for Allvar Gullstrand in 1911 for works on dioptrics of the eye. Wiad Lek. 1967;20(14):1417.

5. Timoney PJ, Breathnach CS. Allvar Gullstrand and the slit lamp 1911 Ir J Med Sci. 2013;182(2):301-5.

6. Klyce SD. Computer-assisted corneal topography. High-resolution graphic presentation and analysis of keratoscopy. Invest Ophthalmol Vis Sci. 1984; 25(12):1426-35.

7. Maldonado MJ, Nieto JC, Piñero DP. Advances in technologies for laser-assisted in situ keratomileusis (LASIK) surgery. Expert Rev Med Devices. 2008;5(2):209-29.

8. Ambrósio Jr R, Caiado AL, Guerra FP, Louzada R, Roy AS, Luz A, et al. Novel pachymetric parameters based on corneal tomography for diagnosing keratoconus. J Refract Surg. 2011;27(10):753-8.

9. Piñero DP, Nieto JC, Lopez-Miguel A. Characterization of corneal structure in keratoconus. J Cataract Refract Surg. 2012;38(12):2167-83.

10. Piñero DP. Technologies for anatomical and geometric characterization of the corneal structure and anterior segment: a review. Semin Ophthalmol. 2015;30(3):161-70

11. Ambrósio Jr R, Valbon BF, Faria-Correia F, Ramos I, Luz A. Scheimpflug imaging for laser refractive surgery. Curr Opin Ophthalmol. 2013;24(4):310-20.

12. Goldberg AV, Tarjan RE. Efficient maximum flow algorithms. Commun ACM. 2014;57(8):82-9.

13. Maldonado MJ, Nieto JC, Díez-Cuenca M, Piñero DP. Repeatability and reproducibility of posterior corneal curvature measurements by combined scanning-slit and placido-disc topography after LASIK. Ophthalmology. 2006;113(11):1918-26.

14. Montalbán R. Caracterización y validación diagnóstica de la correlación de la geometría de las dos superficies de la córnea humana. Alicante: Universidad de Alicante; 2013.

15. Rand RH, Howland HC, Applegate RA. Mathematical model of a Placido disk keratometer and its implications for recovery of corneal topography. Optom Vis Sci. 1997;74(11):926-30.

16. Karpecki PM. Bausch \& Lomb Orbscan II/IIz anterior segment analysis system. In: Wang M, editor. Corneal topography in the wavefront era. Thorofare: Slack Inc.; 2006.

17. Fernandez-Garcia P, Cervino A, Quiles-Guinau L, Albarran-Diego C, GarciaLazaro S, Sanchis-Gimeno JA. Corneal thickness differences between sexes after oxybuprocaine eye drops. Optom Vis Sci. 2015;92(1):89-94.

18. Merklinger HM. Focusing the view camera: a scientific way to focus the view camera and estimate depth of field. Canada: Paperback; 1993.

19. Cheng AC, Rao SK, Lam DS. Accuracy of Orbscan II in the assessment of posterio curvature in patients with myopic LASIK. J Refract Surg. 2007;23(7):677-80.

20. Nawa Y, Masuda K, Ueda T, Hara Y, Uozato H. Evaluation of apparent ectasia of the posterior surface of the cornea after keratorefractive surgery. J Cataract Refract Surg. 2005;31(3):571-3.

21. Dubbelman M, Sicam VA, Van der Heijde GL. The shape of the anterior and posterior surface of the aging human cornea. Vision Res. 2006;46(6-7):993-1001.

22. Piñero DP, Saenz González C, Alió JL. Intraobserver and interobserver repeatability of curvature and aberrometric measurements of the posterior corneal surface in normal eyes using Scheimpflug photography. J Cataract Refract Surg. 2009;35(1):113-20

23. Nasser CK, Singer R, Barkana Y, Zadok D, Avni I, Goldich Y. Repeatability of the Sirius imaging system and agreement with the Pentacam HR. J Refract Surg. 2012;28(7):493-7.

24. Savini G, Barboni P, Carbonelli M, Hoffer KJ. Repeatability of automatic measurements by a new Scheimpflug camera combined with Placido topography. J Cataract Refract Surg. 2011;37(10):1809-16.
25. Salouti R, Nowroozzadeh MH, Zamani M, Fard AH, Niknam S. Comparison of anterior and posterior elevation map measurements between 2 Scheimpflug imaging systems. J Cataract Refract Surg. 2009:35(5):856-62

26. Kanellopoulos AJ, Asimellis G. Forme Fruste Keratoconus Imaging and Validation via Novel Multi-Spot Reflection Topography. Case Rep Ophthalmol. 2013:4(3):199-209.

27. Kanellopoulos AJ, Asimellis G. Clinical Correlation between Placido, Scheimpflug and LED Color Reflection Topographies in Imaging of a Scarred Cornea. Case Rep Ophthalmol. 2014;5(3):311-7.

28. Klijn S, Reus NJ, Sicam VA. Evaluation of keratometry with a novel Color-LED corneal topographer. J Refract Surg. 2015;31(4):249-56.

29. Read SA, Collins MJ, Carney LG, Franklin RJ. The topography of the central and peripheral cornea. Invest Ophthalmol Vis Sci. 2006;47(4):1404-15.

30. Kasprzak HT, Robert ID. Approximating ocular surfaces by generalised conic curves. Ophthalmic Physiol Opt. 2006;26(6):602-9.

31. Turuwhenua J. Corneal surface reconstruction algorithm using Zernike polynomial representation: Improvements. J Opt Soc Am A Opt Image Sci Vis. 2007;24(6):1551-61.

32. Read SA, Collins MJ, Iskander DR, Davis BA. Corneal topography with Scheimpflug imaging and videokeratography: Comparative study of normal eyes. J Cataract Refract Surg. 2009;35(6):1072-81.

33. Alkhaldi W, Iskander DR, Zoubir AM. Model-order selection in zernike polynomial expansion of corneal surfaces using the efficient detection criterion. IEEE Trans Biomed Eng. 2010;57(10):2429-37.

34. Espinosa J, Mas D, Pérez J, Roig AB. Open-access operating algorithms for commercial videokeratographer and improvement of corneal sampling. Appl Opt. 2013;52(7):C24-9.

35. Hong B, Liu W, Li F, Ding X, Yang L. Metrological study on test methods for corneal topographer. Proceedings of 2013 IEEE 11th International Conference on Electronic Measurement and Instruments, ICEMI 2013. 2013.

36. Polette A, Auvinet E, Mari $J$, Brunette I, Meunier J. Construction of a mean surface for the variability study of the cornea. Proceedings - Conference on Computer and Robot Vision, CRV 2014. 2014.

37. Auvinet E, Meunier J, Ong J, Durr G, Gilca M, Brunette I. Methodology for the construction and comparison of 3D models of the human cornea. Proceedings of the Annual International Conference of the IEEE Engineering in Medicine and Biology Society, EMBS. 2012.

38. Zheng S, Ying J, Wang B, Xie Z, Huang X, Shi M. Three-dimensional model for human anterior corneal surface. J Biomed Opt. 2013;18(6):065002.

39. Alonso-Caneiro D, Iskander DR, Collins MJ. Estimating corneal surface topography in videokeratoscopy in the presence of strong signal interference. IEEE Trans Biomed Eng. 2008;55(10):2381-7.

40. Alonso-Caneiro D, Szczesna-Iskander DH, Iskander DR, Read SA, Collins MJ. Application of texture analysis in tear film surface assessment based on videokeratoscopy. J Optom. 2013;6(4):185-93.

41. Alkhaldi W, Iskander DR, Zoubir AM, Collins MJ. Enhancing the standard operating range of a Placido disk videokeratoscope for corneal surface estimation. IEEE Trans Biomed Eng. 2009:56(3):800-9.

42. Huang J, Savini G, Chen H, Bao F, Li Y, Chen H, et al. Precision and agreement of corneal power measurements obtained using a new corneal topographer OphthaTOP. PLoS One. 2015;10(1):e109414.

43. Wang $\mathrm{O}$, Savini G, Hoffer KJ, Xu Z, Feng $Y$, Wen D, et al. A Comprehensive Assessment of the Precision and Agreement of Anterior Corneal Power Measurements Obtained Using 8 Different Devices. PLoS One. 2012;7(9):e45607.

44. Belin MW, Khachikian SS, McGhee CN, Patel D. New technology in corneal imaging. Int Ophthalmol Clin. 2010;50(3):177-89.

45. Montés-Micó R, Cerviño A, Ferrer-Blasco T, García-Lázaro S, Madrid-Costa D. The tear film and the optical quality of the eye. Ocul Surf. 2010;8(4):185-92.

46. Roh HC, Chuck RS, Lee JK, Park CY. The effect of corneal irregularity on astigmatism measurement by automated versus ray tracing keratometry. Medicine (Baltimore). 2015;94(13):e677.

47. Buey Salas MA, Peris MC. Biomecánica y Arquitectura Corneal. Spain: ELSEVIER; 2014

48. Hamano T. Lacrimal duct occlusion for the treatment of dry eye. Semin Ophthalmol. 2005;20(2):71-4.

49. Gomes JA, Tan D, Rapuano CJ, Belin MW, Ambrósio Jr R, Guell UL et al Global consensus on keratoconus and ectatic diseases. Cornea. 2015;34(4):359-69.

50. McGhee CN, Kim BZ, Wilson PJ. Contemporary Treatment Paradigms in Keratoconus. Cornea. 2015;34 Suppl 10:S16-23. 
51. Zadnik K, Barr JT, Edrington TB, Everett DF, Jameson M, McMahon TT, et al. Baseline findings in the Collaborative Longitudinal Evaluation of Keratoconus (CLEK) Study. Invest Ophthalmol Vis Sci. 1998;39(13):2537-46.

52. Wilson SE, Lin DT, Klyce SD. Corneal topography of keratoconus. Cornea. 1991;10(1):2-8.

53. Prisant O, Legeais JM, Renard G. Superior keratoconus. Cornea. 1997;16(6):693-4

54. Weed KH, McGhee CN, MacEwen CJ. Atypical unilateral superior keratoconus in young males. Cont Lens Anterior Eye. 2005;28(4):177-9.

55. Schlegel Z, Hoang-Xuan T, Gatinel D. Comparison of and correlation between anterior and posterior corneal elevation maps in normal eyes and keratoconus-suspect eyes. J Cataract Refract Surg. 2008;34(5):789-95.

56. Montalbán R, Piñero DP, Javaloy J, Alió JL. Scheimpflug photography-based clinical characterization of the correlation of the corneal shape between the anterior and posterior corneal surfaces in the normal human eye. J Cataract Refract Surg. 2012:38(11):1925-33.

57. Montalbán R, Piñero DP, Javaloy J, Alio JL. Correlation of the corneal toricity between anterior and posterior corneal surfaces in the normal human eye. Cornea. 2013;32(6):791-8

58. Montalbán R, Alio JL, Javaloy J, Piñero DP. Correlation of anterior and posterior corneal shape in keratoconus. Cornea. 2013;32(7):916-21.

59. Tomidokoro A, Oshika T, Amano S, Higaki S, Maeda N, Miyata K. Changes in anterior and posterior corneal curvatures in keratoconus. Ophthalmology. 2000;107(7):1328-32.

60. Smolek MK, Klyce SD, Hovis JK. The Universal Standard Scale: proposed improvements to the American National Standards Institute (ANSI) scale for corneal topography. Ophthalmology. 2002;109(2):361-9.

61. Ambrósio Jr R, Nogueira LP, Caldas DL, Fontes BM, Luz A, Cazal JO, et al. Evaluation of corneal shape and biomechanics before LASIK. Int Ophthalmol Clin. 2011;51(2):11-38.

62. Rabinowitz YS. Tangential vs sagittal videokeratographs in the "early" detection of keratoconus. Am J Ophthalmol. 1996;122(6):887-9.

63. Chan JS, Mandell RB, Burger DS, Fusaro RE. Accuracy of videokeratography for instantaneous radius in keratoconus. Optom Vis Sci. 1995;72(11):793-9.

64. Szczotka LB, Thomas J. Comparison of axial and instantaneous videokeratographic data in keratoconus and utility in contact lens curvature prediction. CLAO J. 1998;24(1):22-8.

65. de Sanctis U, Loiacono C, Richiardi L, Turco D, Mutani B, Grignolo FM. Sensitivity and specificity of posterior corneal elevation measured by Pentacam in discriminating keratoconus/subclinical keratoconus. Ophthalmology. 2008;115(9):1534-9.

66. Khachikian SS, Belin MW. Posterior elevation in keratoconus. Ophthalmology. 2009;116(4):816. 816. e1; author reply 816-7.

67. Emre S, Doganay S, Yologlu S. Evaluation of anterior segment parameters in keratoconic eyes measured with the Pentacam system. J Cataract Refract Surg. 2007;33(10):1708-12.

68. Alió JL, Piñero DP, Alesón A, Teus MA, Barraquer Rl, Murta J, et al. Keratoconus-integrated characterization considering anterior corneal aberrations, internal astigmatism, and corneal biomechanics. J Cataract Refract Surg. 2011;37(3):552-68.

69. Ambrósio Jr R, Alonso RS, Luz A, Coca Velarde LG. Corneal-thickness spatial profile and corneal-volume distribution: tomographic indices to detect keratoconus. J Cataract Refract Surg. 2006;32(11):1851-9.

70. Saad A, Gatinel D. Topographic and tomographic properties of forme fruste keratoconus corneas. Invest Ophthalmol Vis Sci. 2010;51(11):5546-55.

71. Smolek MK, Klyce SD. Current keratoconus detection methods compared with a neural network approach. Invest Ophthalmol Vis Sci. 1997;38(11):2290-9.

72. Albertazzi R. Queratocono: pautas para su diagnóstico y tratamiento. Buenos Aires: Ediciones Científicas Argentinas; 2010.

73. Dingeldein SA, Klyce SD, Wilson SE. Quantitative descriptors of corneal shape derived from computer-assisted analysis of photokeratographs. Refract Corneal Surg. 1989;5(6):372-8.

74. Maeda N, Klyce SD, Smolek MK, Thompson HW. Automated keratoconus screening with corneal topography analysis. Invest Ophthalmol Vis Sci. 1994:35(6):2749-57.

75. Holladay JT. Corneal topography using the Holladay Diagnostic Summary. J Cataract Refract Surg. 1997;23(2):209-21.

76. Rabinowitz YS, McDonnell PJ. Computer-assisted corneal topography in keratoconus. Refract Corneal Surg. 1989:5(6):400-8.

77. Calossi A. The optical quality of the cornea. In: Caimi F, Brancato R, editors The aberrometers: theory, clinical and surgical applications. Canelli: Fabiano Editore; 2003.
78. Carney LG, Mainstone JC, Henderson BA. Corneal topography and myopia. A cross-sectional study. Invest Ophthalmol Vis Sci. 1997;38(2):311-20.

79. Piñero DP, Alió JL, Aleson A, Escaf Vergara M, Miranda M. Corneal volume, pachymetry, and correlation of anterior and posterior corneal shape in subclinical and different stages of clinical keratoconus. J Cataract Refract Surg. 2010;36(5):814-25.

80. Cavas-Martínez F, Fernández-Pacheco DG, De La Cruz-Sánchez E, Nieto Martínez J, Fernández Cañavate FJ, Vega-Estrada A, et al. Geometrical custom modeling of human cornea in vivo and its use for the diagnosis of corneal ectasia. PLoS One. 2014;9(10):e110249.

81. Amsler M. The "forme fruste" of keratoconus. Wien Klin Wochenschr. 1961;73:842-3.

82. Toprak I, Yaylali V, Yildirim C. A combination of topographic and pachymetric parameters in keratoconus diagnosis. Cont Lens Anterior Eye. 2015;38(5):357-62.

83. Rabinowitz YS, Rasheed K. KISA\% index: a quantitative videokeratography algorithm embodying minimal topographic criteria for diagnosing keratoconus. J Cataract Refract Surg. 1999;25(10):1327-35.

84. Chastang PJ, Borderie VM, Carvajal-Gonzalez S, Rostène W, Laroche L. Automated keratoconus detection using the EyeSys videokeratoscope. J Cataract Refract Surg. 2000;26(5):675-83.

85. Belin MW, Ambrósio R. Scheimpflug imaging for keratoconus and ectatic disease. Indian J Ophthalmol. 2013;61(8):401-6.

86. Rabinowitz YS. Videokeratographic indices to aid in screening for keratoconus. J Refract Surg. 1995;11(5):371-9.

87. Abad JC, Rubinfeld RS, Del Valle M, Belin MW, Kurstin JM. Vertical D: a novel topographic pattern in some keratoconus suspects. Ophthalmology. 2007:114(5):1020-6.

\section{Submit your next manuscript to BioMed Central and we will help you at every step:}

- We accept pre-submission inquiries

- Our selector tool helps you to find the most relevant journal

- We provide round the clock customer support

- Convenient online submission

- Thorough peer review

- Inclusion in PubMed and all major indexing services

- Maximum visibility for your research

Submit your manuscript at www.biomedcentral.com/submit

) Biomed Central 